\title{
ALMOST PRINCIPAL ELEMENT LATTICES
}

\author{
C. JAYARAM \\ Department of Mathematics \\ University of Swaziland \\ Kwaluseni Campus \\ $\mathrm{P} \backslash$ Bag Kwaluseni \\ Swaziland, Southern Africa
}

\section{E. W. JOHNSON}

Department of Mathematics

University of Iowa

Iowa City, IA 52242

USA

(Received October 25, 1993 and in revised form February 21, 1994)

\begin{abstract}
In this paper, we investigate $C$-lattices for which every localization is a principal element lattice.
\end{abstract}

KEY WORDS AND PHRASES. Multiplicative lattice, Noether lattice, principal element. 1992 AMS SUBJECT CLASSIFICATION CODES. 06F10, 0605.

Let $L$ be a multiplicative lattice with least element 0 and greatest element I. Assume every element of $L$ is the join of elements of a multiplicatively closed subset $C$ of compact elements of $L$ with $I \in C$. We will call such a lattice a $C$-lattice. By a filter on $C$, we mean a multiplicatively closed subset $F$ of $C$ such that $a \in F$ and $b \in C$ and $a \leq b$ imply $b \in F$. If $L$ is a $C$-lattice, then $L$ can be localized at any filter $F$ on $C$. If $p$ is a prime and $F=\{A \in C \mid A \not \leq p\}$, then $F$ is a filter on $C$. Following tradition, we denote localization at $F$ by $L_{p}$.

In this paper, we investigate $L$ under the assumption that, for every maximal element $m$ of $L, L_{m}$ is a principal element lattice. We call such a multiplicative lattice an almost principal element lattice. We show, for example, that $L$ is an almost principal element lattice if and only if every compact element of $L$ is principal and for every maximal element $m \in L, L_{m}$ satisfies the ascending chain condition. The reader is referred to [1] for general background and terminology.

Let $F$ be a filter on $C$. For $a \in L$, define $a_{F}=\bigvee\{x \in C \mid m x \leq a$, for some $m \in F\}$. Let $L_{F}=\left\{a_{F} \mid a \in L\right\}$. Then $L_{F}$ is a subposet of $L$. Further, under the multiplication of defined on $L_{F}$ by $a_{F} \circ_{F} b_{F}=(a b)_{F}, L_{F}$ is a multiplicative lattice. We denote the meet and join operations in $L_{F}$ by $\wedge_{F}$ and $\vee_{F}$. We note that for $S \subseteq L, \bigvee_{F}\left\{s_{F} \mid s \in S\right\}=(\bigvee S)_{F}$, and 
$\bigwedge_{F}\left\{s_{F} \mid s \in S\right\}=\left(\bigwedge\left\{s_{F} \mid s \in S\right\}\right)_{F}$. For finite sets $S, \bigwedge_{F}\left\{s_{F} \mid s \in S\right\}=\left(\bigwedge\left\{s_{F} \mid s \in S\right\}\right)$. We denote the collection of filters of $C$ by $F^{\prime}(C)$. We observe that for $a, b \in L, a=b$ if and only if $a_{m}=b_{m}$ for every maximal element $m$ of $L$.

Throughout, $L$ is assumed to be a $C$-lattice.

LEMMA 1. The product of compact elements of $L$ is compact.

PROOF. Let $K^{\prime}$ and $K^{\prime \prime}$ be compact elements of $L$. Write $K=E_{1} \vee \cdots \vee E_{m}$ and $K^{\prime \prime}=$ $E_{1}^{\prime} \vee \cdots \vee E_{n}^{\prime}$, where $E_{\imath}, E_{\jmath}^{\prime} \in C$. Then $K K^{\prime \prime}=\bigvee_{\imath, \jmath} E_{\imath} E_{\jmath}^{\prime}$, and since $C$ is multiplicatively closed, $K K^{\prime \prime}$ is compact.

LEMMA 2. If $L$ is an almost principal element lattice, then every compact element of $L$ is principal and $L$ is an $r$-lattice.

PROOF. $L$ is compactly generated. Let $k$ be a compact element. Then for every maximal clement $m$ of $L, k_{m}$ is principal in $L_{m}$. Hence, for all $a \in L, a_{m} \wedge_{m} k_{m}=\left(a_{m}: m k_{m}\right)_{o_{m}} k_{m}$. As $k$ is compact, this gives $(a \wedge k)_{m}=\left(a_{m}: k_{m} k_{m}\right)_{\circ_{m}} k_{m}=(a: k)_{m \circ_{m}} k_{m}=((a: k) k)_{m}$. Similarly, $(a k: k)_{m}=(a k)_{m}: k_{m}=\left(a_{m} \circ_{m} k_{m}\right):_{m} k_{m}=a_{m} \vee_{m}\left(0_{m}: k_{m}\right)=(a \vee(0: k))_{m}$. It follows that $k$ is weak principal, and hence principal [1, Proposition 1.1].

Recall that in an $r$-lattice, principal elements are compact, and the set of compact elements is multiplicatively closed. Recall also that a multiplicative lattice $L$ satisfies the weak union condition if for all $a, b, c \in L$ with $a \not \leq b$ and $a \not z c$, there exists a principal element $e \leq a$ with $e \not \leq b$ and $e \not z c$.

COROLLARY 1. If $L$ is an almost principal element lattice, then $L$ satisfies the weak union condition.

PROOF. In a $C$-lattice, given $a \not \leq b$ and $a \not \leq c$, there exists $e \in C$ satisfying $e \leq a$, with $e \not \leq b$ and $e \not z c$.

We also have the following.

LEMMA 3. If $L$ satisfies the weak union condition, then $\mathrm{L}$ is an $r$-lattice.

PROOF. It suffices to show that elements of $C$ are principally generated. Hence, assume $k \in C$. Let $s$ be the supremum of all principal elements $e \leq k$. If $s<k$ then $k \not s$ and $k \not s$ so there exists a principal element $e \leq k$ such that $e \not s s$. This contradicts the definition of $s$ and shows that $k$ is principally generated.

LEMMA 4 [3, Lemma 0]. Let $L$ be a local principal element lattice with maximal element $m$. Then $L$ is every element $a \neq 0$ of $L$ is a power of $m$ (with $m^{0}=I$ ). Hence, $L$ is a chain and therefore distributive.

COROLLARY 2. Let $L$ be an almost principal element lattice. Then $L$ is distributive.

PROOF. For $a, b, c \in L,(a \wedge(b \vee c))_{m}=((a \wedge b) \vee(a \wedge c))_{m}$ for every maximal element $m \in L$. Hence $a \wedge(b \vee c)=(a \wedge b) \vee(a \wedge c)$.

LEMMA 5. Let $L$ be a distributive quasi-local lattice. If $L$ satisfies the weak union condition, then every compact element is principal.

PROOF. The proof is similar to that of [3, Lemma 3].

LEMMA 6. If $L$ is distributive and satisfies the weak union condition, then for every filter $F \in L(C), L_{F}$ is distributive and satisfies the weak union condition. 
PROOF. If $L$ is distributive, then clearly $L_{F}$ is distributive. The remainder of the lemma follows from the observation that if $e \in L$ is principal, then $e$ is compact and $e_{F} \in L_{F}$ is principal.

The following Theorem 1 gives a condition equivalent to the hypothesis of Lemma 6 .

THEOREM 1. Let $L$ be a $C$-lattice. Then the following are equivalent.

1. $L$ is distributive and satisfies the weak union condition.

2. Every compact element is principal.

PROOF. Suppose every compact element is principal. Then [5, Proposition 5$] L$ is distributive, and clearly $L$ satisfies the weak union condition.

Now, assume that $L$ is distributive and satisfies the weak union condition. Then for every prime $p$ of $L, L_{p}$ is a quasi-local, distributive and satisfies the weak union condition. Let $a \in L$ be compact. Then for every maximal element $m$ of $L, a_{m}$ is compact and hence (Lemma 4) principal.

As a consequence, we have the following, where by a Noether lattice, we mean a noetherian $r$-lattice (i.e., an $r$-lattice satisfying the ascending chain condition).

COROLLARY 3. $L$ is a distributive Noether lattice satisfying the weak union condition if and only if every element is principal.

It is obvious that every principal element lattice is an almost principal element lattice but the converse is not true. For example, let $R$ be either an almost Dedekind domain or an almost multiplication ring (see, for example, [4]). Then the lattice $L(R)$ of ideals of $R$ is an almost principal element lattice which but need not be Noetherian. Therefore $L(R)$ need not be a principal element lattice.

The following Theorem 2 gives a characterization of almost principal element lattices.

THEOREM 2. $L$ is an almost principal element lattice if and only if every compact element of $L$ is principal and $L$ is locally noetherian.

PROOF. Assume $L$ is an almost principal element lattice. Clearly $L$ is locally noetherian. If $k \in L$ is compact, then $k$ is principal (Lemma 2).

Now, assume that every compact element of $L$ is principal and that $L$ is locally noetherian. By Theorem 1, $L$ is distributive and satisfies the weak union condition. Then for each maximal element $m \in L, L_{m}$ is a distributive quasi-local lattice satisfying the weak union condition. Every element of $L_{m}$ is compact, so (Theorem 1) every element of $L_{m}$ is principal.

LEMMA 7. Let $L$ be a totally ordered quasi-local $r$-lattice with maximal element $m$. If $m \neq m^{2}$ then $m$ is principal.

PROOF. Let $e$ and $f$ be principal elements $\leq m$ and $\not m^{2}$. We can assume $f \leq e$. Then either $f=e$ or $f \leq m e$. The latter contradicts $f \not m^{2}$,so $f=e$. Now, let $g \leq m$ be any principal element. If $g \not \leq m^{2}$ then $g=e$. Otherwise, $g \leq m^{2} \leq e$. As $m$ is principally generated, it follows that $m=e$.

LEMMA 8. Let $L$ be a local Noether lattice with maximal element $m$. Then $L$ is totally ordered if and only if the interval $\left[\mathrm{m}^{2}, m\right]$ is totally ordered, and this is the case if and only if $m$ is principal.

PROOF. If $L$ is totally ordered, then clearly the interval $\left[m^{2}, m\right]$ is totally ordered. Let $e_{1}, \ldots, e_{n}$ be principal elements with join $m$. Choose $e_{\imath} \vee m^{2}$ maximal in $\left\{e, \vee m^{2}\right\}_{j=1}^{n}$. By Nakayama's Lemma, if follows that $m=e_{2}$. Hence $m$ is principal. 
Now, assume that the interval $\left[\mathrm{m}^{2}, m\right]$ is totally ordered. As in the previous paragraph, $m$ is principal. On the other hand, if $m$ is principal, then by Lemma 5, every element $a \neq 0$ of $L$ is a power of $m$. Hence $L$ and $\left[m^{2}, m\right]$ are totally ordered.

We note that in a local Noether lattice with maximal element $m$, the interval $\left[m^{2}, m\right]$ is totally ordered if and only if there are no elements strictly between $m^{2}$ and $m$.

THEOREM 3. Let $L$ be a $r$-lattice. Then $L$ is an almost principal element lattice if and only if $L$ is locally noetherian and for every maximal element $m \in L$, the interval $\left[m^{2}, m\right]$ is totally ordered.

PROOF. Assume $L$ is an almost principal element lattice. Let $m$ be a maximal element of $L$. Then $L_{m}$ is a Noether lattice, and $m_{m}$ is principal, so (Lemma 8) the interval $\left[m_{m}^{2}, m_{m}\right]=\left[m^{2}, m\right]$ is totally ordered.

Now assume that for every maximal element $m, L_{m}$ is a Noether lattice and $\left[m_{m}^{2}, m_{m}\right]=$ $\left[m^{2}, m\right]$ is totally ordered. Then (Lemma 8) $m_{m}$ is principal in $L_{m}$ and so every element $a \neq 0$ in $L_{m}$ is a power of $m_{m}$. It follows that every element of $L_{m}$ is principal, and hence that $L$ is an almost principal element lattice.

THEOREM 4. Let $L$ be an $r$-lattice. Then $L$ is an almost principal element lattice if and only if $L$ is locally noetherian and for every maximal element $m$ of $L$, every $m$-primary element of $L$ is a power of $m$.

PROOF. Assume $L$ is an almost principal element lattice. Let $m$ be a maximal element of $L$. Then $m_{m}$ is principal in $L_{m}$ and (Theorem 2) $L_{m}$ is a Noether lattice. Let $q$ be a $p$-primary element. Then $q_{m}$ is a $m_{m}$-primary element in $L_{m}$ and (Lemma 5) $q_{m}$ is a power of $m_{m}$, say $q_{m}=m_{m}^{n}$. As both $q$ and $m^{n}$ are $m$-primary, it follows that $q=m^{n}$. The converse follows from Theorem 3 .

We summarize our main results.

THEOREM 5. Let $L$ be a $C$-lattice. The the following are equivalent.

1. $L$ is an almost principal element lattice.

2. $L$ is locally noetherian and every compact element of $L$ is principal.

3. $L$ is locally noetherian, satisfies the weak union condition and is distributive.

4. $L$ is locally Noether and for every maximal element $m$, the interval $\left[m^{2}, m\right]$ is totally ordered.

5. $L$ is locally Noether and for every maximal element $m$, the interval $\left[m^{2}, m\right]$ is simple or trivial.

6. $L$ is locally Noether and for every maximal element $m$, every $m$-primary element is a power of $m$.

\section{REFERENCES}

1. ANDERSON, D. D. Abstract commutative ideal theory without chain condition, Algebra Universalis $\underline{6}$ (1976), 131-145

2. DILWORTH, R. P. Abstract commutative ideal theory, Pacific J. Math. $\underline{12}$ (1962), 481-498

3. JOHNSON, E. W., and LEDIAEV, J. P. Representable distributive Noether lattices, Pacific J. Math. $\underline{28}$ (1969), 561-564

4. LARSON,. M. D., and MCCARTHY, P. J. Multiplicative theory of ideals, Academic Press, New York, 1971

5. MCCARTHY, P. J. Arithmetical rings and multiplicative lattices, Ann. Math. Pure Appl. $\underline{82}$ (1969), 267-274 


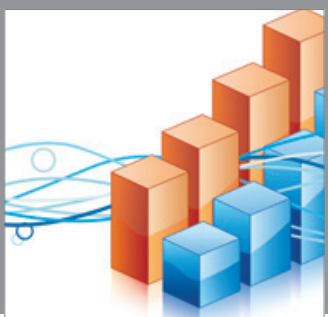

Advances in

Operations Research

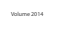

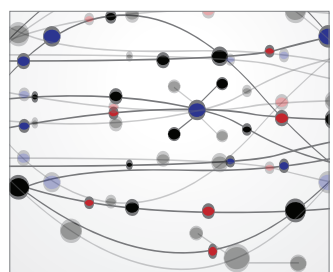

\section{The Scientific} World Journal
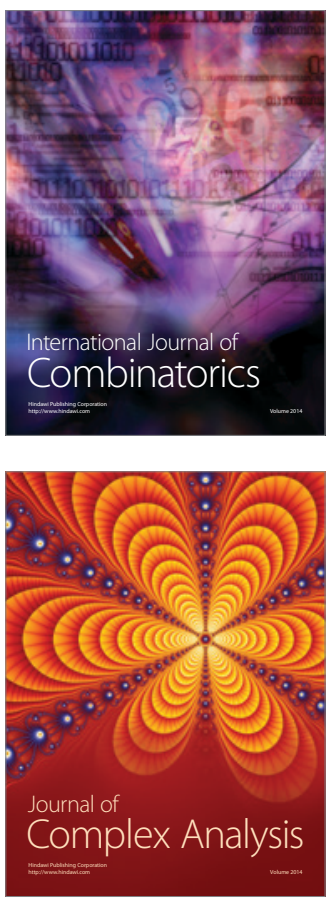

International Journal of

Mathematics and

Mathematical

Sciences
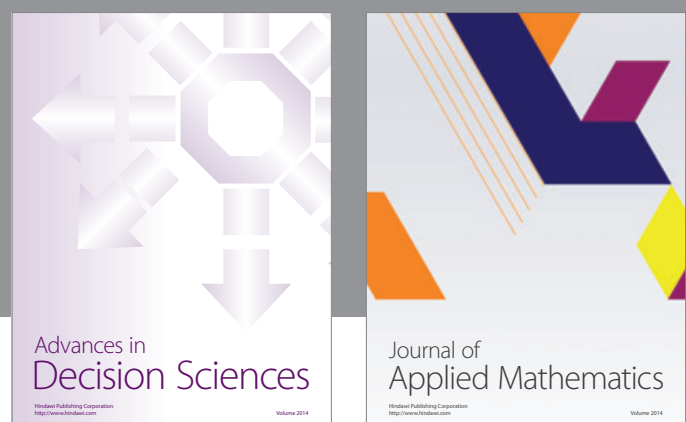

Journal of

Applied Mathematics
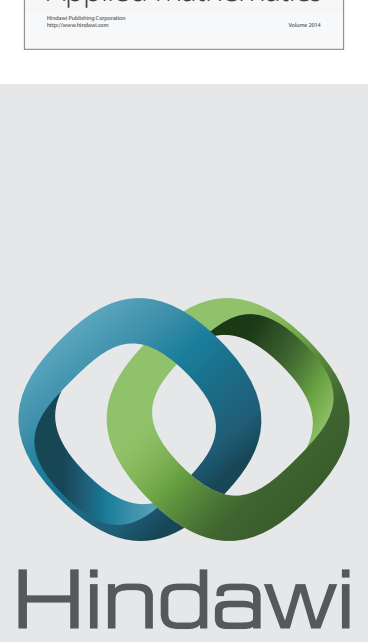

Submit your manuscripts at http://www.hindawi.com
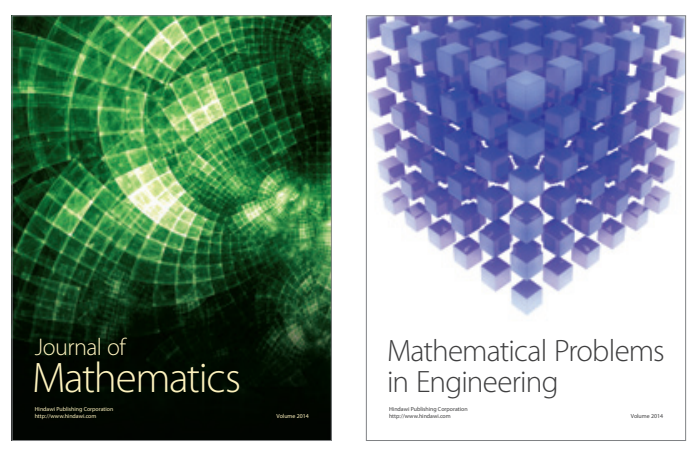

Mathematical Problems in Engineering
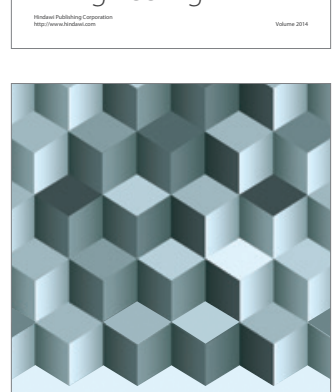

Journal of

Function Spaces
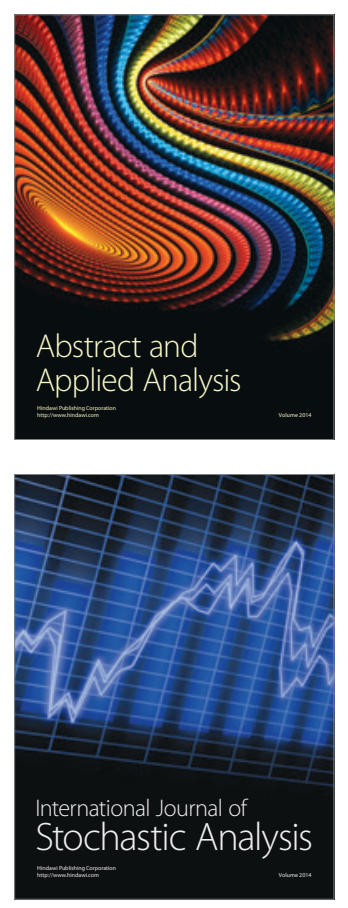

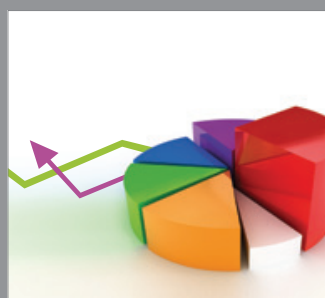

ournal of

Probability and Statistics

Promensencen
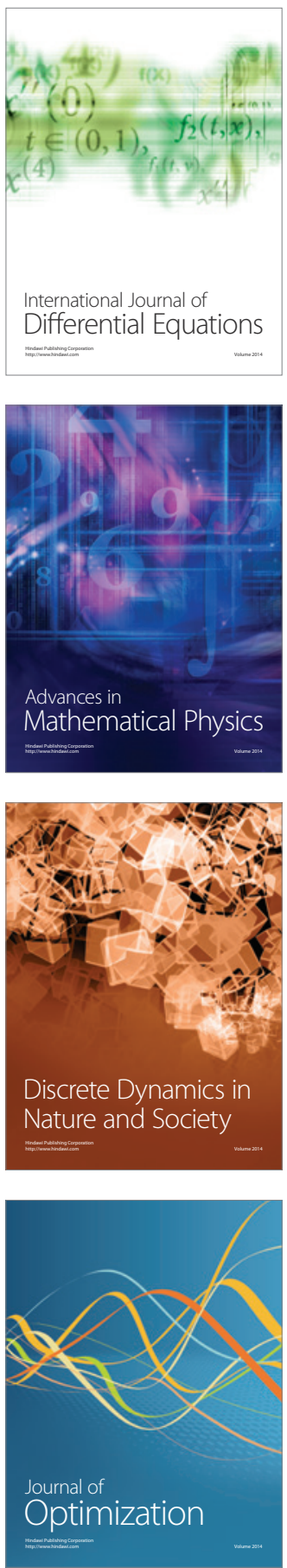